American Journal of Immunology 1 (1): 37-41, 2005

ISSN 1553-619X

(c) 2005 Science Publications

\title{
Effects of $50 \mathrm{HZ}$ Magnetic Field on Some Factors of Immune System in the Male Guinea Pigs
}

\author{
${ }^{1}$ H.Sedghi, ${ }^{2}$ S.Zare, ${ }^{2}$ H.Hayatgeibi, ${ }^{2}$ S.Alivandi and ${ }^{3}$ A.G.Ebadi \\ ${ }^{1}$ Department of Physics, Urmia University,Urmia, Iran \\ ${ }^{2}$ Department of Biology,Urmia University,Urmia, Iran \\ ${ }^{3}$ Department of Biology,Islamic Azad University of Sari,Sari branch 48164-194, Iran
}

\begin{abstract}
This study investigated the bio-effects of $50 \mathrm{~Hz}$ MF exposure on some Immunological factors (ACTH, cortisol and glucose levels) in male Guinea pigs. The capacity of $50 \mathrm{~Hz}$ Electromagnetic Fields (EMF) to interfere in the weakness of endocrine system has been a relevant fact into the scientific community, since we are more susceptible for this kind of exposure, in modern days. The 60-day-old Guinea pigs, which average of weight was 350-400 g, the Guinea pigs were divided in two groups, one of them was the control group while the other one suffered interference of a variable EMF of $50 \mathrm{~Hz}$, intensity $0.207 \mu \mathrm{T}, 4$ hours a day, for a 5 consecutive he results were collected by the weight comparison before and after of the exposure period, as well as the cortisol and glucose levels. These facts are strongly linked with the immunological system. After 5 days, the animals were anaesthetized and had their blood withdrew from their hearts. The samples produced serum where ACTH (quimiluminescent method), cortisol (radioimunoassay method) and glucose (oxidative method) levels were evaluated. The results showed that $50 \mathrm{~Hz}$ EMF acted on rats without any significant weight change, but affecting ACTH,cortisol and glucose levels in a significant way, 33.06\% ( $\mathrm{p}=0.033) ; 27.00 \%(\mathrm{p}=0.004)$ and $56.23 \%(\mathrm{p}=0.014)$ respectively when were compared with the control group. For this reason, EMF is able to change the serum levels of ACTH, cortisol and glucose, possible changing the endocrinological regulations.
\end{abstract}

Key words: ACTH, magnetic field, cortisol, glucose, weight, guinea pigs

\section{INTRODUCTION}

Over the past few years, considerable attention has been given to the potential bio-effects of magnetic field (MF). Epidemiological studies have suggested that MF may increase the risk of various types of cancer, including leukemia, brain and breast tumours. The characteristic biological effects of MF appear to be functional changes in the central nervous system, endocrine and immune systems ${ }^{[1]}$.

Electromagnetic fields became increasingly common constituent of the general and workplace environments early in the $20^{\text {th }}$ century, but some lifestyles and occupations are associated with more than the average amount of exposure to electromagnetic fields. May patterns of exposure in the general and workplace environments be able to be identified with increased intensity and duration of exposure to electromagnetic field. The exaggerated rise on Na rates, K-ATPase, when the organism is subjected to EMF of $60 \mathrm{~Hz}$, results in the increase of stress level on population subjected to this kind of produced radiation, mainly, by the proper home electrics or by workplace environment ${ }^{[2]}$. There is conflicting evidence about whether exposure to ELF fields can directly alter the structure of DNA and chromatin. Most reviews have concluded that mutational and neoplasic transformation effects are not expected to happen ${ }^{[3,4]}$. However, some reports suggest that ELF fields may cause direct effects on the DNA ${ }^{[5]}$. Even so, the population is not free of the low frequency EMF action, radiated from household appliances ${ }^{[6]}$.

At the last decade, there have been many studies of the possible relation between different kinds of cancer, like the child leukaemia, brain tumours, lung cancer, breast cancer and lymphomas, among others and residential exposure to ELF magnetic fields ${ }^{[7-9]}$. Different studies reported epidemiological studies about the risks of cancer related to exposure to EMF $(50 / 60 \mathrm{~Hz})$ from powerlines ${ }^{[3,4,10-12]}$. Similar studies reported the risk of adverse effects on human reproduction associated to $\mathrm{EMF}^{[4,13-15]}$. In later, more rigorous studies, Chernoff et $a l^{[13]}$ reported significant early pregnancy loss from exposure to residential $50 \mathrm{~Hz}$ magnetic fields, but low numbers in the high-exposure group limit conclusions from this study. The truth is that, if it won't possible to confirm this correlation, it's not also conclusive the fact of that the electromagnetic waves are harmless when fall on live organisms, between a specific potency and frequency. Several reports have shown that exposure of rodents to ELF magnetic fields reduces the production, secretion or both of melatonin from the pineal gland ${ }^{[16]}$. Exposure of rats for 42 days to circularly polarised magnetic fields $(0,02$ and $0,1 \mu \mathrm{T})$ reduced both day time and night time melatonin levels by $20-25 \%{ }^{[17,18]}$

The reduction on melatonin secretion and the increase on the ACTH and cortisol release are suggestive that the exposed builds to low frequency electromagnetic waves are

Corresponding Author: Zare, S., Department of Biology, Faculty of Sciences, Urmia University, Urmia, Iran 
more liable to high levels of stress and consequently, more liable to develop illness or organic imbalance. Dumanshii ${ }^{[19]}$ presented the first study indicating that environmental exposure to power frequency (50 and 60 $\mathrm{Hz}$ ), electric and magnetic fields (EMF) might increases the risk of chronic disease-in this case mortality-from cancer in children. Three years later this study was followed by a report by Milham on the relation between cancer and work in occupations with presumed exposure to EMF. These original studies have been followed by a substantial number of subsequent reports on various cancer diseases. To a certain extent these reports have confirmed the original findings, in particular with respect to childhood leukaemia ${ }^{[11]}$. The established mode of interaction between EMF and humans is by means of induced currents and sufficiently strong fields may result in acute health effects. Nevertheless, the epidemiological research has gradually expanded and has also come to include outcomes other than cancer. This review focuses on neurodegenerative diseases and on suicide and depression ${ }^{[1]}$. A study on Alzheimer's disease ${ }^{[20]}$ reported an apparent three-fold increase in risk for magnetic-field exposure. The increase was attributable to a limited number of occupations that typically receive high exposure levels, such as swing machine operators. Sobel ${ }^{[20]}$ has also reported an association for amyotrophic lateral sclerosis. These finding need further examination and follow up. A several number of neurodegenerative diseases, including cancer, become difficult to characterise. It is observed that the most difficult thing resides on the impossibility of registering the neurological injuries, cancer and other appeals and their caused connection, owing to they seems smaller than should be, because they don't demand any compulsory notification $^{[5,21-23]}$.

\section{MATERIALS AND METHODS}

Animals: Male Guinea pigs (Pasteur Institute, Iran) weighing $350-400 \mathrm{~g}$ at the time of experiments were housed at $25^{\circ} \mathrm{C}$ in a cage under a $12-12 \mathrm{~h}$ light/dark cycle, with free access to food and water. Treated Guinea pigs $(n=12)$ were exposed to MF $(0.207 \mu \mathrm{T}-50 \mathrm{~Hz}$; 4h/day) for 5 consecutive days. Control and treated animals were sacrificed under light anesthesia (halothane $2.5 \%$, in air).

Exposure system: The control group A $(n=15)$ and the exposed group B $(n=15)$ were housed in six standardised cages (five animals per cage) with $120 \mathrm{~cm}$ length, $90 \mathrm{~cm}$ width and $50 \mathrm{~cm}$ height, put on supports made in polystyrene with $135 \mathrm{~cm}$ width, $110 \mathrm{~cm}$ length and $135 \mathrm{~cm}$ height, every cages located in the same room. The group B, was exposed to EMF of $50 \mathrm{~Hz}$, senoidal, of $0.207 \mu \mathrm{T}$, measured by a gaussimeter, on the scale of $0-100 \mathrm{mG}$, during four hours a day, being one hour in the morning from 8:00h to 10:00h and one hour in the afternoon from $14: 00 \mathrm{~h}$ to $16: 00 \mathrm{~h}$, for 5 consecutive days. It was used, as radiation fonts, transformers of 220/110 volts of $500 \mathrm{VA}$, working with opened secondary and controlled by an electronic timer, connected to a tension estabilizator.
Control animals were accomplished by simply leaving the transformers from the polystyrene supports. The transformers, put on under the polystyrene supports, were previously examined and tested at Departament of BiologyUniversity of Urmia to confirm their working parameters. The electromagnetic field was measured inside the cage and it kept constant independent of the position. It should be noted that, except for the weekly cages cleaning, the rats were not moved or handled during this experiment. Exposed animals should be compared with control that have been derived from the same source and simultaneously handled and assayed in the same way, except for the presence of the fields. The animals were weighed at the first day experiment and at the 5th day using a mechanic balance. On the terminal day of the experiment, at the 6th day, all animals were anaesthetised with ethylic ether. After that, the blood was taken by a cardiac puncture ( $3 \mathrm{~mL}$ ) to obtain the serum, always at the same time in the morning, from 8 to $9 \mathrm{~h}$.

Metabolic assessment: Serum concentration of ACTH was measured by quimiluminescent reaction, using the ACTH Immulit kit, DPC (USA) and the Immulit equipment, DPC (USA). Serum concentration of cortisol was measured by radioimunoassay technique (RIE) using the cortisol coat-A-count kit, DPC (USA) and the analysis was made in a gama meter of scintillation, auto-gamma, CobraII (Packard a Camberra Company), at the Departamento de Fisiologia da UFPE. Serum glucose levels were measured by the glucose oxidase method using the PAPglucose kit-Labtest Diagnostic-Iran. Every measure was done duplicated.

Statistical analysis: Data were analyzed using Stat SPSS software (Version 12). The results were expressed as means \pm SEM and comparison of two means was made using Student's t-test. A value of $\mathrm{p}<0.05$ was considered to be significant.

\section{RESULTS}

Physical weight: The results show that the initial body weight average of control group $(n=15)$ was $351.33 \pm 0.23 \mathrm{~g}$ and the final average was $364.23 \pm 0.54 \mathrm{~g}$, while the initial body weight average of exposed group $(n=15)$ was $385.34 \pm 1.34 \mathrm{~g}$ and the final average was $399.45 \pm 0.12 \mathrm{~g}$. The Fig.1, shows that exposure to electromagnetic radiation doesn't cause any significant alteration in body weight when compared to control group.

Cortisol concentration: The results showed that the average of control group $(n=15)$ was $4.33 \pm 0.02 \mathrm{pg} \mathrm{mL}^{-1}$ while the average of exposed group $(n=15)$ was $5.78 \pm 0.11 \mathrm{~g}$ The cortisol serum concentration, after 5 exposure days, at the exposed group, presented an increase of $27 \%$ group B when compared to group $\mathrm{A}$, in a significant way $(\mathrm{p}=0.004)$, Fig. 2. $\left(\times 10^{-4}\right)$.

Glucose serum concentration: In relation to glucose serum concentration after the exposure to electromagnetic radiation, results showed that the average of control group 


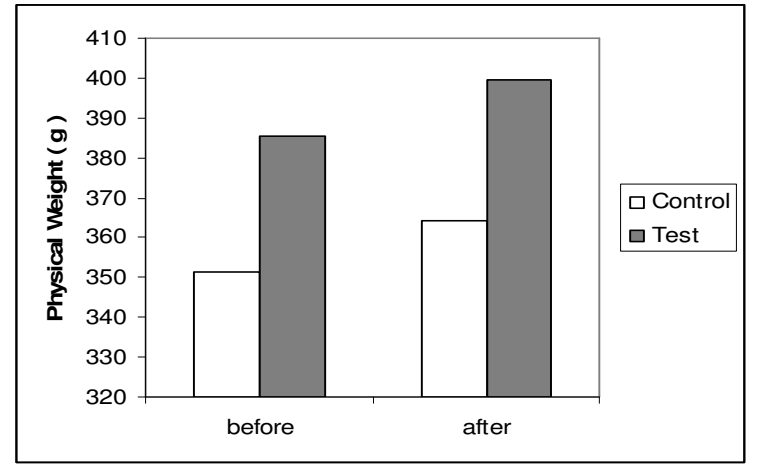

Fig. 1: Determination of body weight, in $\mathrm{g}$, of control animals and exposed animals to electromagnetic (test), before and after 5 exposure days. The results obtained are the average \pm standard detour (SD). $\mathrm{N}=15, \mathrm{p}=0.021$

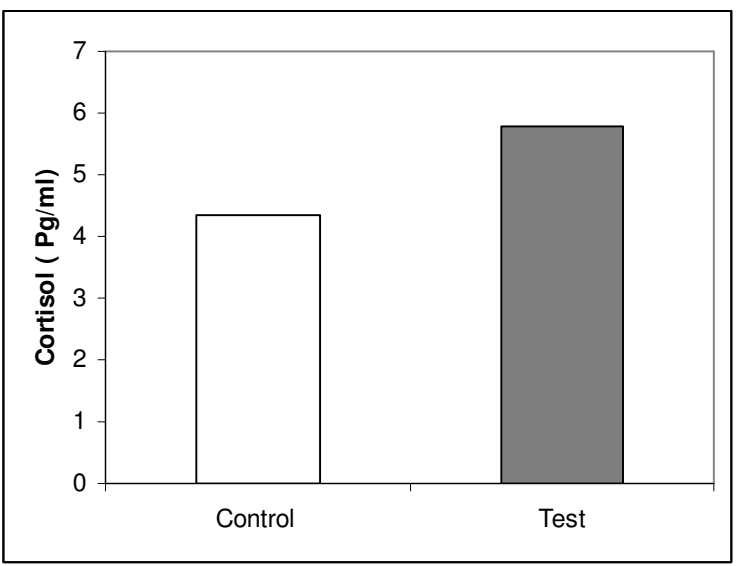

Fig. 2: Determination of cortisol serum concentration in pg $\mathrm{mL}^{-1}$ of control animals and exposed animals to EMF. The results obtained are the average \pm standard detour (SD). $\mathrm{N}=15 \mathrm{p}=0.004$

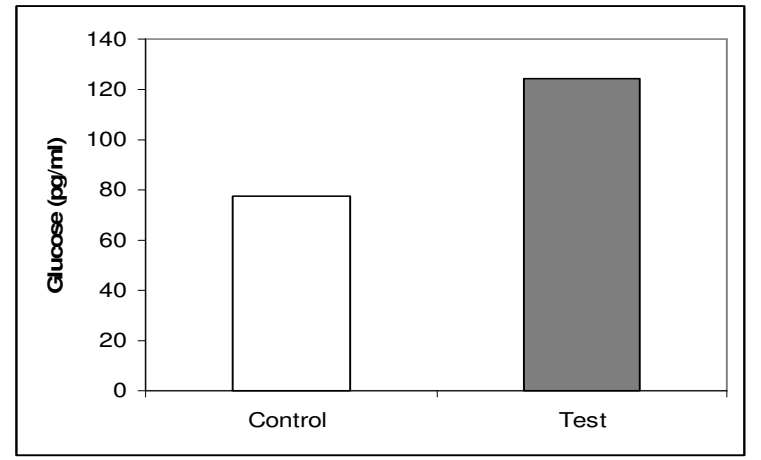

Fig. 3: Determination of glucose serum concentration in $\mathrm{mg} \mathrm{dL^{-1 }}$ of control animals and exposed animals. The results obtained are the average \pm standard deviation (SD). $\mathrm{N}=15 \mathrm{p}=0.014$

$(\mathrm{n}=15)$ was $77.66 \pm 9.32 \mathrm{mg} \mathrm{\textrm {dL } ^ { - 1 }}$ while the average of exposed group $(n=15)$ was $124.16 \pm 8.05$ and at the exposed group it was observed an increase of $56.23 \%$, when compared to control group, in a significant way $(\mathrm{p}=0.014)$, Fig. 3. $\left(\times 10^{-4}\right)$

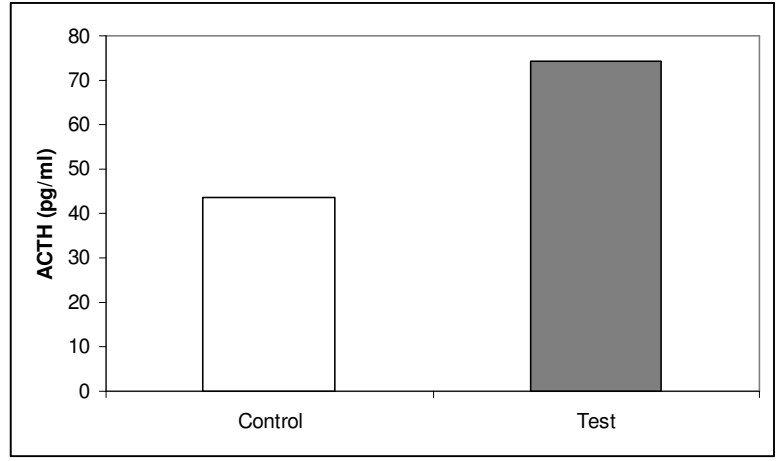

Fig. 4: Determination of ACTH serum concentration in pg $\mathrm{mL}^{-1}$ of control animals and exposed animals to EMF. The results obtained are the average \pm standard deviation (SD). $\mathrm{N}=15 \mathrm{p}=0.033$

ACTH level: The Fig. 4 shows that the exposure to electromagnetic radiation causes an increase of more than 33. $06 \%$ in ACTH level of group B $(n=15)$ compared to group A. It is observed that the average of control group $(\mathrm{n}=15)$ was $45.33 \pm 1.66 \mathrm{pg} \mathrm{mL}^{-1}$ while the average of exposed group $(n=15)$ was $77.23 \pm 5.23(n=15)$, presenting a degree of significance of $\mathrm{p}=0.033$.

\section{DISCUSSION}

Male normal Guinea pigs were exposed to EMF of 50 $\mathrm{Hz}$, senoidal of $0.207 \mu \mathrm{T}$, four hours a day for 4 days. The animals were maintained in normal conditions of lighting (day and night), environment temperature varying from $26^{\circ}$ to $30^{\circ} \mathrm{C}$ and with water and food ad libittum. The exposed animals presented lifelong similar to the control animals, as well as the body weight average of exposed group is comparable to control group in this study. The choice of intensity of $0.207 \mu \mathrm{T}$ was because of the necessity in using a higher intensity than in residences and most work-places, the average $50 / 60 \mathrm{~Hz}$ magnetic fields are between 0,1 and $0,3 \mu \mathrm{T}^{[3]}$, so that it was used a value ten times higher than the maximum value expected, by the fact of that depending on the distance between the conductors and ground and magnetic flux densities can average $22 \mu \mathrm{T}$, depending on the current load in the line ${ }^{[23]}$ and this value could depend on also the geographic location of the kind of magnetic material near the area .

In our experiment the animals subjected to EMF didn't present any difference, statistically significant, in relation to the body weight when compared to the animals of control group, in agreement with Bonhomme-Fraive's study ${ }^{[18]}$ which reports that Swiss mice, continuously exposed to ELF of $50 \mathrm{~Hz}$ and $50 \mu \mathrm{T}$ for 350 days, at different study times, there was no significant difference in body weight between the exposed and unexposed animals. However, Wilson et al. ${ }^{[25]}$ shows that exposure of SL (short-light, 8h light: $16 \mathrm{~h}$ dark) animals to a steady state $60 \mathrm{~Hz}$ magnetic field for 3h/day for 42 days resulted in a statistically significant reduction in body weight, compared to shamexposed SL animals. Nevertheless, it seems to us that this 
reduction in body weight is more associated with the absence of light than the exposure to EMF. The reason for measuring the cortisol serum concentration in this experiment was because the cortisol is well known as being a stress indicator ${ }^{[26,27]}$. In our study was observed an increase statistically significant in cortisol serum concentration at the exposed animals when compared to control group, the same occurring with the ACTH serum levels, indicating that the exposure to EMF of $50 \mathrm{~Hz}$, in spite of being considered a low frequency, it's capable to stress the organism. This result is in agreement with Tenforde's study ${ }^{[4]}$, when exposure of monkeys produced an increase in urinary corticoids which lasted about 6 days, after which the corticoid levels returned to baseline despite continued exposure to the field. In another study, a similar effect on corticoids in rats persisted for 4 months ${ }^{[19]}$. Our results also confirm the Stevens' hypothesis ${ }^{[12]}$ that suggests that field exposure have effects not on melatonin, but also on the reproductive hormones and the immune system. In other way, some reports indicate that acute exposure for one night to a linearly polarized magnetic field at $10 \mu \mathrm{T}$ has no effect on hormonal or immune parameters in healthy male volunteers ${ }^{[28]}$. This result couldn't be used as a parameter to our studies, because they work with acute exposure, only one night, while our animals were exposed for 29 consecutive days, characterising a chronic exposure or stress. According to Radon et al. ${ }^{[27]}$, human exposure to EMF doesn't cause any alteration on cortisol concentration, however, once again, it's difficult to compare with our findings, because at this study it was used high frequency EMF and for much shorter time.

In our study the serum glucose concentration also had a significant increase on the exposed group when compared to control group, in accordance with Armario ${ }^{[6]}$ and Marti and Armario ${ }^{[26]}$, which shows that repeated stress is associated with the sensitivity of glucose, inducing hyperglycaemia. Obtained data in this experiment make us to believe that the EMF of $60 \mathrm{~Hz}$, in spite of being a low frequency EMF, activates the hypothalamic-hipophyseadrenal axis with consequent increase on cortisol and glucose release what characterises an immune depression patient's condition of immune system. In spite of the obtained data confirm our point of view and many published papers', we believe that many experiments are still necessary, with the purpose of explaining which frequency, intensity, exposure time and other parameters involved with the EMF, to protect us from harms that life can cause to us ${ }^{[24,25,29-32]}$.

\section{ACKNOWLEDGMENTS}

Thanks are due to Colleagues in Faculty of Sciences (Urmia University) for their excellent technical assistance.

\section{REFERENCES}

1. Ahlbom, A., 2001. Neurodegenerative diseases, suicide and depressive syntoms in relation to emf.bioelectromagnetics supplement. Stockolm, Sweden, 5: 132-143.
2. Blank, M., L. Soo and V. Papstein, 1995. Efeito dos campos magnéticos de baixa frequiência na atividade do Na, K-ATPase. Bioelectrochemi. Bioenerg., 38: 267-273.

3. National Radiobiological Protection Board, 1994b. Electromagnetic fields and risk of cancer: Supplementary report by the Advisory Group on Nonionizing Radiation of 12 April 1994. Radiol. Prot. Bill., 154: 10-12.

4. Tenforde, T.S., 1996. Interaction of ELF Magnetic Fields with Living System. In: Polk, C. and Postow, E., Eds. Biological Effects of Electromagnetic Fields. Boca Raton, FL: CRC Press, pp: 185-230.

5. Lai, H. and N.P. Singh, 1997. Melatonin and a spintrap compound blocked radiofrequency, radiation induced DNA strand breaks in rat brain cells. Bioelectromagnetics, 18: 446-454.

6. Armario, A., J. Marti and M. Gil, 1990. The serum glicose response to acute estresse is sensitive to the intensity of the stressor and to habituation. Psychoneuroendocrinology, 15: 341-347.

7. Balcer, K. and K. Elizabeth, 1995. Gene expression flowing 60-Hz magnetic field exposure. Enviromental Health Perspectives, Boston, MA, pp: 18-22, June.

8. Erren, T.C., 1997. Epidemiologic studies of EMG and breast cancer risk: A biologically based overview. In: The melatonin hypothesis: breast cancer and the use of electric power. Columbus: Battelle Press.

9. Loomis, D.P., D.A. Savitz and C.V. Ananth, 1994. Breast cancer mortality among female electrical workers in the United States. J. Natl. Cancer Inst., 86: 885-886.

10. Savitz, D.A., 1993. Overview of epidemiological research on electric and magnetic fields and cancer. Am. Ind. Hyg. Ass. J., 54: 197-204.

11. Health, C.W., 1996. Electromagnetic field exposure and cancer: a review of epidemiological evidence. Cancer J. Clin, 46: 29-44.

12. Stenvens, R.G. and S. Davis, 1996. The melatonin hypotheses: electric power and breast cancer. Environ. Health Persp., 104: 135-140.

13. Chernoff, N., J.M. Rogersm and R. Kavet, 1992.A review of the literature on potential reproductive and developmental toxicity of electric and magnetic fields. Toxicology, 74: 91-126.

14. Brent, R.L., D.A. Beckman and C.P. Landel, 1993. Clinical teratology. Curr. Opin. Pediatr., 5:201-211.

15. Shaw, G.W. and L.A. Croen, 1993. Human adverse reproductive outcomes and electromagnetic fields exposures: Review of epidemiologic studies. Environ. Health Persp., 101: 107-119. 
16. Repacholi, M.H. and B. Greenebaum, 1999. Interaction of static and extremely low frequency electric and magnetic fields with living systems: Health effects and research needs. Bioelectromagnetics, 20: 133-160.

17. Bonhomme-Faiver, L., A. Mace, Y. Bezie, S. Marion, G. Bindoula, A.M. Szekely, N. Frenois, H. Auclair, O.S. Arbouys and E. Bizi, 1998. Alterations of biological parameters in mice chronically exposed to low-frequency $(50 \mathrm{~Hz})$ electromagnetic fields. Life Sci., 62: 1271-1280.

18. Kato, M., K.I. Homna, T. Shigemitsu and Y. Shiga, 1991. Effects of exposure to a circularly polarized, 50 $\mathrm{Hz}$ magnetic fields on melatonin levels in rats. Bioelectromagnetics, 14: 97-106.

19. Dumanshii, U.D., 1976. Hygienic evaluation of electromagnetic field generated by high-voltage power lines. Gigyena I Sanitariya, 8: 19-23.

21. De Boer, S.F., S.J. Koopmans, J.L. Slangen and V.J.Gugten,1990. Plasma catecholamine, corticosterone and glucose responses to repeated estresse in rats: Effect of interstressor interval length. Physiol. Behav., 47: 1117-1124.

20. Sobel, E., 1996. Eletromagnetic field exposure may cause increased production of amyloid beta and eventually lead to Alzheimer`s disease (hypothesis). Neurology, 47: 1594-1600.

22. Lerchl, A., K.O. Nonaka and R.J. Reiter, 1997 . Marked rapid alterations in nocturnal pineal serotonin metabolism in mice and rats exposed to weak intermittent magnetic fields. Biochem. Biophys. Res. Commun., 169: 102-108.

23. Loescher, W., M. Mevissen, W. Lechmacher and A. Stamm, 1993. Tumor promotion in a breast cancer model by exposure to a weak alternating magnetic field. Cancer Lett., 71: 75-81.

24. Simon, N.J., 1992. Biological effects of static magnetic fields: A review. International Cryogenic Materials Comission, Boulder, Colorado, pp: 284.
25. Wilson, B.W., K.S. Matt, J.E. Morris, L.B. Sasser, D.L. Miller and E.A. Larry, 1999. Effects of $60 \mathrm{~Hz}$ magnetic field exposure on the pineal and hypothalamic-pituitary-gonadal axis in the siberian hamster (Phodopus sungorus). Bioelectromagnetics, 20: 224-232.

26. Marti, O. and A. Armario, 1997. Influence of exposure to chronic estresse on the pattern of habituation of pituitary-adrenal hormones, prolactin and glucose. Stress, 1: 179-189.

27. Radon, K., D. Parera, D.M. Rose, D. Jung and L. Vollrath , 2001. No effects of pulsed radio frequency electromagnetic fields on melatonin, cortisol and selected markers of the Immune system in man. Bioelectromagnetics, 22: 280-287.

28. Selmaoui, B., A. Bogdan, A. Auzeby, J. Lambrozo and Y. Touitou, 1996b. Acute exposure to $50 \mathrm{~Hz}$ magnetic field does not affect hematologic or immunologig functions in healthy young men. Bioelectromagnetics, 17: 364-372.

29. Selmaoui, B., J. Lambrozo and Y. Touitou, 1997. Endocrine functions in young men exposed for one night to a $50 \mathrm{~Hz}$ magnetic field. A circadian study of pituitary, thyroid and adrenocortical hormones. Life Sci., 61: 473-486.

30. Welker, H.A., P. Semm, R.P. Willing, J.C. Commentz, W. Iltschko and L. Vollrath, 1983.Effects of an artificial magnetic field on serotonin $\mathrm{N}$-acetyl transferase activity and melatonin content of the rat pineal gland. Exp Brain Res., 50: 426-432.

31. Wilson, B.W., L.E. Anderson, D.I. Hilton and R.D. Phillips,1981. Chronic exposure to $60-\mathrm{Hz}$ electric fields: Effects on pineal function in the rat. Bioelectromagnetics, 2: 371-380.

32. Yellon, S.M., 1996. Acute $60 \mathrm{~Hz}$ magnetic field exposure effects on the melatonin rhythm in the pineal gland and circulation of the adult Djungarian hamster. J. Pineal. Res., 16: 136-144. 